\title{
Factores asociados a irritabilidad en adultos con episodio depresivo en Lima Metropolitana.
}

Factors associated to irritability in adults with depressive episode in Metropolitan Lima.

\author{
Yesenia M. Salazar-Saavedra ${ }^{1, \text { a, b }}$, Javier E. Saavedra-Castillo ${ }^{1, c, d ; 2, e}$.
}

\section{RESUMEN}

Objetivo: Determinar factores sociodemográficos y clínicos asociados a la presencia de irritabilidad en adultos con episodio depresivo (ED) en Lima Metropolitana. Materiales y Métodos: Estudio observacional, correlacional, transversal, utilizando la base de datos de adultos desde los 18 años del Estudio Epidemiológico de Salud Mental de Lima Metropolitana y Callao del año 2012, en una muestra probabilística multietápica de 4445 adultos. Los instrumentos usados fueron una ficha de datos sociodemográficos, MINI (Entrevista Neuropsiquiátrica Internacional), Cuestionario de Salud Mental de Colombia (modificado), Índice de Calidad de Sueño de Pittsburgh, EMBU modificado y Cuestionario de Experiencias Tempranas Adversas. Se utilizó estadística descriptiva, regresión simple y multivariada para estimar la prevalencia de adultos con irritabilidad en episodio depresivo (IED) y determinar la asociación entre IED y variables independientes. Resultados: La prevalencia de IED fue de 34,9\%. Se halló asociación estadísticamente significativa con: menor edad de inicio del ED, mayor severidad del ED, presencia de trastorno de ansiedad generalizada, abuso/dependencia de alcohol, algunos síntomas depresivos (autorreproches, problemas de concentración e indicadores suicidas), conductas violentas, menor calidad de sueño, estilos de crianza de sobreprotección y favoritismo, y presencia de eventos adversos antes de los 18 años. Conclusiones: Varias características y factores diferencian la depresión con irritabilidad de la que carece de este síntoma. La posibilidad de que la irritabilidad llegue a ser considerada como un especificador del episodio depresivo, contribuiría a desarrollar estrategias para su más precisa identificación y manejo clínico.

PALABRAS CLAVE: Episodio depresivo, irritabilidad, adultos.

\section{SUMMARY}

Objective: To determine factors associated to the presence of irritability in adults with depressive episode (DE) in Metropolitan Lima in 2012. Materials and methods: Database of the Epidemiological Study of Metropolitan Lima and Callao, carried out in 2012, was used to make an observational, correlational, cross-sectional study. A multistage probabilistic sample of 4445 adults of 18 years and older. The instruments were: MINI (International Neuropsychiatric Interview), Mental Health Questionnaire of Colombia (modified), modified brief EMBU, a questionnaire of adverse early experiences, Pittsburgh Sleep Quality Index and a sociodemographic data sheet. Descriptive statistics, simple and multivariate regression were used to estimate prevalence of adults with irritability in depressive episode (IDE) and to determine the association between IDE and independent variables. Results: The prevalence of IDE was $34.9 \%$. A statistically significant association was found with: early age of onset of

Facultad de Medicina Alberto Hurtado, Universidad Peruana Cayetano Heredia. Lima, Perú.

2 Oficina de Apoyo a la Investigación y Docencia Especializada, Instituto Nacional de Salud Mental "Honorio Delgado-Hideyo Noguchi”. Lima, Perú.

a Bachiller en Medicina, ${ }^{\mathrm{b}}$ Médico-Cirujano; ${ }^{\mathrm{c}}$ Médico-Psiquiatra; ${ }^{\mathrm{d}}$ Profesor;

e Director Ejecutivo. 
$\mathrm{DE}$, greater severity of $\mathrm{DE}$, presence of generalized anxiety disorder (GAD), abuse/dependence on alcohol, some depressive symptoms (such as self-reproach, concentration problems and suicidal indicators), violent behaviors, a lower quality of sleep, parenting styles of overprotection and favoritism, and the presence of adverse events before the age of 18. Conclusions: The possibility that irritability may be considered as a specifier of the depressive episode, would contribute to the development of strategies for its identification and clinical management.

KEY WORDS: Depressive episode, irritability, adults.

\section{INTRODUCCIÓN}

La depresión representa un problema importante de salud pública, considerada por la Organización Mundial de la Salud (OMS) como la primera causa mundial de discapacidad $(1,2)$, y pronosticada como primera causa de discapacidad en países en vías de desarrollo para el año 2020 (3). Asimismo, en otros países de Latinoamérica, como Brasil y Chile, la prevalencia del trastorno depresivo es más alta en comparación con otras psicopatologías (4). En el Perú, los estudios realizados por el Ministerio de Salud (MINSA) han demostrado que las enfermedades neuropsiquiátricas, encabezadas por la depresión, poseen la mayor carga de enfermedad, seguidas de las lesiones no intencionales, condiciones perinatales y enfermedades cardiovasculares (5).

Su importancia clínica dentro de la atención primaria se relaciona a comorbilidades tales como el hipotiroidismo, enfermedades cardiovasculares, trastornos de sistema nervioso central, el dolor crónico, y condiciones inflamatorias como la diabetes y obesidad $(6,7)$. Por lo tanto, un inadecuado manejo o la no identificación causan un grave impacto en la vida de las personas, aumentando el ausentismo laboral, y reduciendo la calidad de vida, la capacidad funcional, la productividad, la integración social e independencia de éstas (8).

Con relación a la descripción de los síntomas clínicos, la Clasificación Internacional de Enfermedades (CIE-10) define al episodio depresivo (ED) como un trastorno del humor caracterizado por un decaimiento del ánimo, anhedonia, fatigabilidad, baja autoestima, sentimientos de culpa, pensamientos suicidas, problemas de concentración, agitación o inhibición psicomotriz, y alteración del sueño y apetito. Este trastorno se clasifica en leve, moderado, o severo, dependiendo de la cantidad de síntomas que la persona presenta en un periodo mínimo de 2 semanas. Además, hay ausencia de síntomas hipomaniacos y maniacos, y el episodio no se atribuye al consumo de sustancias psicoactivas o a algún trastorno mental orgánico (9).

La irritabilidad es un síntoma frecuentemente descrito en un ED; sin embargo, éste no está incluido dentro de los criterios de clasificación de este trastorno del ánimo en adultos. Este síntoma se describe como una respuesta emocional provocada por un estímulo que fácilmente lleva al estado de ira (10), considerándose una expresión frustrante de la no-recompensa, que se traduce como una reacción al bloqueo de realización de metas (11). Asimismo, la manifestación de la depresión puede verse enmascarada por la irritabilidad, así como el consumo excesivo de alcohol, el comportamiento histriónico, la exacerbación de fobias o síntomas obsesivos preexistentes, o por preocupaciones hipocondriacas.

Algunos estudios (10-16) han reportado que la irritabilidad es una de las características que se asocian a un mayor grado de severidad de la depresión, a intentos suicidas, a una baja calidad de vida y a otras comorbilidades. A pesar de ello, la reciente revisión del Manual Diagnóstico y Estadístico de Trastornos Mentales (DSM-5) sólo la ha considerado como posible síntoma en niños y adolescentes, mas no en adultos, por lo que podría ser ignorado por los médicos que siguen los síntomas estándar. Además, se ha informado que la irritabilidad ocurre entre un tercio y la mitad de los pacientes con depresión mayor, siendo parte de un fuerte factor principal de dicho trastorno (12). Es por ello que actualmente se sugiere una mayor consideración entre las descripciones clínicas del ED en el adulto $(13,14)$.

Así mismo, la irritabilidad ha sido descrita como síntoma en varios desórdenes mentales, tales como el trastorno de ansiedad generalizada, trastorno de estrés postraumático, trastorno limítrofe de la personalidad, trastorno antisocial de la personalidad, síndrome de abstinencia, ludopatía, y trastorno psicoafectivo (15). Además, ha sido asociada a condiciones psiquiátricas crónicas como el trastorno de déficit 
Factores asociados a irritabilidad en adultos con episodio depresivo en Lima Metropolitana.

de atención con hiperactividad, retardo mental y la enfermedad de Alzheimer; trayendo consecuencias no favorables para el individuo (16). De igual manera, ha sido frecuentemente usada como una característica diagnóstica para diferenciar a la depresión bipolar de la unipolar $(13,14)$.

Existen estudios en los que se evaluó la presencia de irritabilidad, los cuales fueron realizados en ambientes clínicos, que implicó únicamente la participación de pacientes con trastorno depresivo. Por ejemplo, en el estudio de Perlis et al., que incluyó a 2307 pacientes con trastorno depresivo mayor sin eventos psicóticos, se reportó que el $46 \%$ presentó irritabilidad al menos la mitad del tiempo durante la semana previa al estudio; prevaleciendo este síntoma en mujeres jóvenes, desempleadas, de estrato social bajo, y que reportaron al menos un intento suicida. Además, la presencia de irritabilidad estuvo correlacionada a la severidad del cuadro depresivo. Por otro lado, en este estudio se mencionó que la ira o sus manifestaciones, como la irritabilidad, pueden ser considerados factores de riesgo para enfermedades cardiovasculares (17).

Un estudio prospectivo realizado por Judd et al. en el año 2013, en centros de salud de Estados Unidos consistió en determinar la prevalencia de irritabilidad o cólera y sus efectos en el curso de un ED de 536 pacientes. Como resultado, se obtuvo que el 54,5\% de pacientes presentó irritabilidad o cólera durante el curso del ED, y que este síntoma incrementaba significativamente en relación de la severidad y mayor duración del cuadro clínico, falta de control de impulsos, mayores tasas de abuso de sustancias y trastorno de ansiedad, trastorno de la personalidad antisocial, reducción de la satisfacción con la vida, y antecedentes familiares de trastorno bipolar (18).

Solo dos estudios de tipo poblacional se han encontrado. Uno de ellos fue un estudio de Fava y colaboradores, que consistió en realizar una encuesta sobre desórdenes mentales, National Comorbidity Survey Replication 'NCS-R', a 9282 personas mayores de 18 años en Estados Unidos, encontrándose que el $27,7 \%$ de participantes diagnosticados con depresión mayor presentó irritabilidad durante el peor episodio alguna vez en su vida. La presencia de dicho síntoma durante el periodo de depresión mayor estuvo asociada a una temprana edad de inicio de los síntomas, la persistencia en el tiempo de éstos, fatiga durante los episodios, trastorno de ansiedad y discapacidad. Además, se halló una mayor prevalencia de casos de depresión mayor con irritabilidad, en mujeres jóvenes entre los 18 y 44 años, desempleadas, con depresión severa, de estrato social bajo y pobre calidad de vida, y que presentaron al menos un intento suicida (19).

El otro estudio poblacional reciente, realizado por Balbuena et al., implicó el uso de la base de datos de la Encuesta de Morbilidad Psiquiátrica del Reino Unido 2000 , realizada a 8338 personas que oscilaban entre los 16 a 74 años, residentes de distintos hogares del Reino Unido. Este estudio consistió en determinar si la incorporación de los síntomas de irritabilidad y ánimo inestable a los criterios de depresión (tanto de la CIE-10 como del DSM-5) constituiría una escala válida para el diagnóstico de este trastorno del ánimo. Las preguntas de irritabilidad no formaban parte de las preguntas de depresión y fueron tomadas del cuestionario sobre trastornos de personalidad. Como resultado, se encontró que tanto la irritabilidad como el ánimo inestable podrían formar parte de los síntomas principales de la depresión mayor, incluyendo el ánimo deprimido y la anhedonia (12). Asimismo, mencionan que la irritabilidad sería un indicador de un curso más severo y crónico de la depresión en mujeres, por lo que sugieren ampliar los estudios en este campo $(12,17-$ 19).

Existe evidencia de patrones de variabilidad en los síntomas de depresión entre países desarrollados y en vías de desarrollo, y se ha reportado la presencia de somatización, alteración del sueño, ideas de culpa, y conducta suicida en algunas sociedades; mientras que en otras sociedades, algunos de estos síntomas pueden estar ausentes, incluso perteneciendo a una misma nación (20-26). Algunas de las causas de identificación de la variabilidad sintomatológica al momento de la consulta médica se debe a la influencia de la cultura de cada sociedad, los idiomas utilizados para reportar sus propias dolencias, las decisiones de cada tratamiento y la relación médico-paciente (20).

En el caso del síntoma de irritabilidad, no existen estudios en nuestro medio y a nivel latinoamericano, por lo que la presente investigación serviría de aporte a la exploración de características y factores que podrían estar asociados a la presencia de irritabilidad en un ED en países en vías de desarrollo, contribuyendo a la información de otros estudios poblacionales. Asimismo, la base de datos del Estudio Epidemiológico de Salud Mental de Lima Metropolitana y CallaoReplicación 2012 (EESMLMC) (27) integró la pregunta sobre irritabilidad como parte de un síntoma adicional de la depresión, lo que permitirá explorar variables asociadas no reportadas, además 
de los factores estudiados en las investigaciones anteriormente mencionadas (12,17-19).

Por lo tanto, con el fin de obtener una mayor claridad sobre el papel que la irritabilidad desempeña dentro de un cuadro depresivo, y así posteriormente poder desarrollar e implementar estrategias que mejoren la identificación y abordaje clínico de este trastorno, el objetivo principal de este estudio fue determinar los factores sociodemográficos, características clínicas del ED, otros trastornos mentales, conductas suicidas y violentas, calidad de sueño, estilos de crianza, y eventos tempranos adversos, que estén asociados a la presencia de irritabilidad en adultos con ED en Lima Metropolitana; adicionalmente se estimará la prevalencia de adultos que presenten irritabilidad dentro del ED, y se evaluará la significancia de las asociaciones anteriormente mencionadas.

\section{MATERIAL Y MÉTODOS}

Se trata de un estudio observacional, correlacional, transversal, obtenido de una base de datos secundaria el Estudio Epidemiológico de Salud Mental de Lima Metropolitana y Callao (EESMLMC), realizado en el año 2012, en el que se halló datos sociodemográficos, socioculturales, así como la presencia de trastornos clínicos, con principal énfasis en la salud mental de la población.

La muestra obtenida del EESMLMC fue de tipo compleja, realizada por medio de un proceso probabilístico de tres etapas: La primera etapa consistió en la selección de conglomerados (100 viviendas contiguas), cuya información fue brindada por el Instituto Nacional de Estadística e Informática (INEI) a partir del Censo del 2007. En la segunda etapa se seleccionó viviendas contiguas de manera aleatoria, mediante un programa estadístico. Y en la tercera etapa se realizó la selección de las personas encuestadas, utilizando la tabla de Kish a excepción de la mujer casada o cohabitante (27).

La población de estudio consistió en 4445 adultos de ambos sexos de 18 años a más, que cumplían con los siguientes criterios de inclusión: que hayan sido residentes habituales (por lo menos $1 \mathrm{mes}$ ) de viviendas particulares de la ciudad de Lima y Callao, y que no tuvieran impedimento de comunicarse verbalmente con el entrevistador. No se incluyó a las personas que residían en los distritos en balnearios del sur de Lima, ni a las personas que tuvieron impedimento de comunicarse verbalmente con el entrevistador (27).
Fueron diversos los instrumentos de medición que fueron sometidos a distintos controles para ser utilizados en las encuestas del EESMLMC, tomando en cuenta la consistencia interna de cada instrumento mediante el modelo alfa de Cronbach. Detalles de los datos psicométricos y de los instrumentos y de las preguntas utilizadas pueden ser encontrados en otra fuente de información (28). Los instrumentos utilizados para este trabajo fueron los siguientes:

Datos demográficos: se incluyó información sociodemográfica basada en preguntas de la Encuesta Nacional de Hogares (ENAHO) del INEI, incluyendo edad, sexo, lugar de nacimiento, tiempo viviendo en Lima, lengua materna, grado de instrucción, estado civil, situación laboral (trabajo remunerado en la última semana y desempleo), información de la vivienda para obtener los indicadores de pobreza según necesidades básicas insatisfechas (NBIs).

MINI: Mini Entrevista Neuropsiquiátrica Internacional (Versión en español, CIE-10): Elaborado por Sheehan, Lecrubier y colaboradores (29). Evaluó la presencia del ED, número de síntomas depresivos, edad de inicio del primer ED, y comorbilidad psiquiátrica. Este instrumento ha sido adaptado en el INSM para obtener prevalencia de vida, anual y actual (27).

Cuestionario de Salud Mental de Colombia (modificado): El cuestionario de Salud Mental en Colombia, fue sometido a un proceso de adaptación y ampliación para uso en nuestro medio por el INSM (30). Las preguntas relacionadas con conductas violentas: "¿Alguna vez le ha pegado a un niño tan fuerte como para hacerle moretones?”; “¿Desde los 18 años, ha estado en más de una pelea que haya acabado a golpes?";" ¿Alguna vez ha usado un arma, como una piedra, un pali, un cuchillo (navaja), o una pistola en una pelea?". Con el objetivo de ampliar la exploración sobre indicadores suicidas se incluyeron preguntas específicas sobre deseo, ideación, planeamiento e intento suicida a través de las preguntas: "¿Alguna vez en su vida ha deseado morir?" (sí=1, no =0); “¿Alguna vez en su vida ha deseado quitarse la vida?" (sí=1, no $=0$ ); “ ¿Alguna vez ha planeado quitarse la vida?" ( $s i ́=1$, no $=0$ ); “ ¿Alguna vez ha intentado hacerse daño o quitarse la vida?" (sí=1, no $=0$ ).

Índice de calidad de sueño de Pittsburgh: Evalúo distintas características de la calidad de sueño, tomando como referencia al estudio realizado por Luna-Solis, Robles-Arana y Agüero-Palacios (31). 


\section{EMBU (Egna Minen Bertraffande Uppfostran} Breve-Modificado): Encuesta que evalúa estilos de crianza parental y eventos adversos antes de los 18 años; deriva en cuatro dimensiones: rechazo, calor emocional, sobreprotección y favoritismo. El estudio original del Instituto Nacional de Salud Mental "Honorio Delgado-Hideyo Noguchi" (INSM "HDHN") incluyó sólo algunas preguntas representativas de cada dimensión en función a la carga factorial del estudio realizado por Someya y colaboradores (32) (tabla 7). Se agregaron preguntas sobre eventos adversos tempranos especificados (tabla 8).

La variable dependiente de estudio fue el síntoma de irritabilidad dentro del ED, y las variables independientes fueron factores sociodemográficos, calidad de sueño, estilos de crianza y eventos adversos tempranos, trastornos mentales, y otros problemas que incluyen conductas suicidas (deseo, pensamiento, planteamiento, e intento suicida), y conductas violentas, descritas anteriormente. Específicamente se evaluó el ED diferenciado en leve, moderado y severo de acuerdo con el número de síntomas según la CIE-10. Así mismo, para evaluar la presencia del síntoma de irritabilidad dentro del ED, se incluyó este síntoma dentro de los criterios del mencionado trastorno con la siguiente pregunta directa: “ ¿Se sentía especialmente irritable (o rabioso), o se molestaba fácilmente la mayor parte del tiempo?". Esta pregunta se circunscribió al periodo de dos semanas o más en las que presentaba sintomatología clínica de depresión (27).

El análisis estadístico se realizó con el programa SPSS 21 para muestras complejas. Todas las prevalencias fueron ponderadas con el fin de obtener una distribución poblacional equitativa en cuanto al sexo y grupo etario. Para estimar las medidas de tendencia central y la prevalencia de adultos que presentaron irritabilidad en ED, se trabajó con estadística descriptiva. Se utilizó el análisis bivariado (regresión simple) para establecer asociación entre la variable dependiente y variables independientes. Para evaluar la relación de la variable dependiente con variables categóricas, se realizaron pruebas chi-cuadrado convertidas al estadístico $\mathrm{F}$ como una variante del estadístico de chi-cuadrado corregido de Rao-Scott de segundo orden y la significación basada en sus grados de libertad y un nivel de significancia de $<0,05$. Para evaluar la asociación con variables numéricas se utilizó el modelo lineal general para muestras complejas. Se realizó análisis multivariados (regresión logística), estimando los valores de OR con un IC95\% y $\mathrm{p}<0,05$ para evaluar la significancia estadística de diversos factores que fueron controlados con las características sociodemográficas y la severidad del ED.

El EESMLMC contó con la aprobación del Comité de Ética Institucional. El presente estudio se realizó previa aprobación de las direcciones de Instituto Nacional de Salud Mental "Honorio Delgado-Hideyo Noguchi" y del Comité de Ética de la Universidad Peruana Cayetano Heredia. Este trabajo de investigación no requirió de la elaboración de un consentimiento informado, por tratarse de una base de datos secundaria. Así mismo, se mantuvo el anonimato de los participantes.

\section{RESULTADOS}

\section{Factores sociodemográficos}

De los 4445 participantes, 842 (18,94\%) adultos presentaron ED alguna vez en la vida, y de estos últimos participantes, el $34,9 \% \quad(n=325)$ presentó irritabilidad dentro del ED (IED) de referencia. Se puede observar que existió mayor prevalencia de IED en los varones $(43,6 \%, \mathrm{OR}=1,308$, IC $95 \%=0,918$ $1,865)$ en comparación de las mujeres $(37,1 \%, \mathrm{OR}=1)$ sin ser estadísticamente significativo (tabla 1). En los análisis bivariados la prevalencia de irritabilidad en ED fue significativamente más alta en el grupo de 18 a 24 años $(46,1 \%$, IC95\%=1,54-4,459), y más baja en adultos de 65 años a más (27,4\%). Sin embargo, no alcanza nivel de significancia en los análisis multivariados al controlarse con las demás variables sociodemográficas. Lo mismo se observa con respecto al estado civil donde se encontró que las personas viudas o divorciadas presentaban significativamente una menor prevalencia en comparación con las solteras en la regresión simple, pero que no se sostiene en el análisis multivariado. Con respecto a la condición educativa no se encontraron diferencias estadísticamente significativas ni por nivel de estudios ni por la presencia de analfabetismo. Tampoco se encontró diferencias significativas según las áreas de residencia ni por el nivel de pobreza entre las personas depresivas con o sin irritabilidad.

La única variable sociodemográfica asociada significativamente fue con personas desocupadas ( $\sin$ trabajo remunerado una semana antes de la encuesta), pues si bien la prevalencia de IED en las personas desocupadas $(39,0 \%$, IC $95 \%=0,742-1,441)$ como no desocupadas $(39,8 \%$, IC $95 \%=0.512-9,654)$ 
Salazar-Saavedra Y, Saavedra-Castillo J.

Tabla 1. Asociación entre irritabilidad y factores sociodemográficos en adultos con ED (CIE-10)a

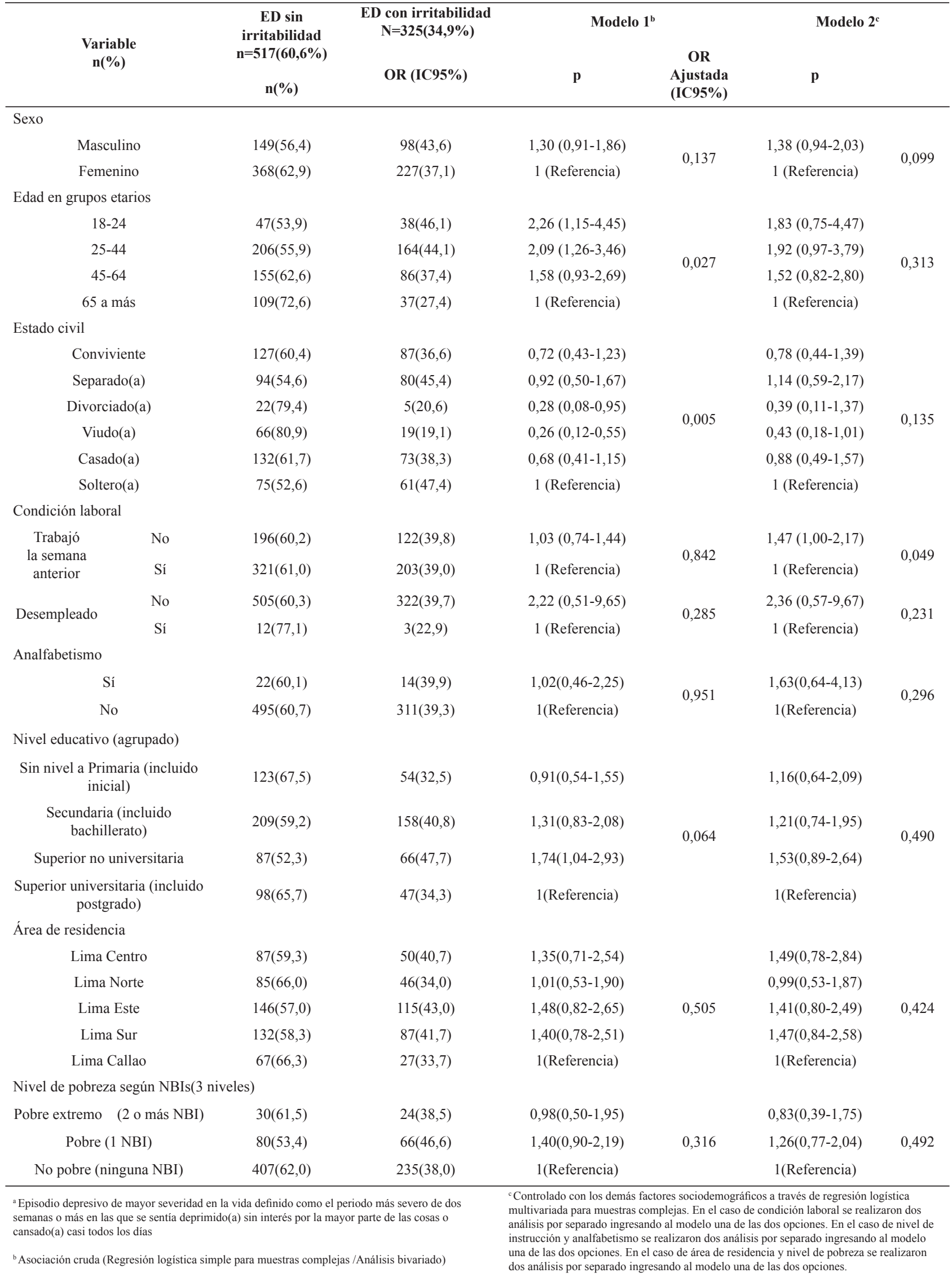


Factores asociados a irritabilidad en adultos con episodio depresivo en Lima Metropolitana.

Tabla 2. Prevalencia y OR de síntoma de irritabilidad en personas con ED (CIE-10 a ) según niveles de severidad y edad de inicio del ED

\begin{tabular}{|c|c|c|c|c|c|c|c|}
\hline \multirow{2}{*}{\multicolumn{2}{|c|}{$\begin{array}{r}\text { Variable } \\
\text { No } \\
\mathbf{n}=\mathbf{5 1 7}\end{array}$}} & \multicolumn{2}{|c|}{ Síntomas de irritabilidad } & \multicolumn{2}{|c|}{ Modelo $1^{\text {b }}$} & \multicolumn{2}{|l|}{ Modelo 2 } \\
\hline & & $\begin{array}{c}\text { Sí } \\
\mathbf{N}=325 \\
\text { Media (ES) }\end{array}$ & $\begin{array}{c}\text { OR } \\
\text { (IC 95\%) }\end{array}$ & $\mathbf{P}$ & $\begin{array}{c}\text { OR } \\
\text { ajustado } \\
\text { (IC95\%) }\end{array}$ & $\mathbf{p}$ & \\
\hline \multicolumn{2}{|c|}{ Edad de inicio } & $32,3(0,85)$ & $27,8(0,89)$ & -- & $<0,001$ & $0,98(0,97-1,00)$ & 0,049 \\
\hline \multicolumn{2}{|c|}{ Prevalencia de vida del ED } & $60,6(2,1)$ & $39,4(2,1)$ & -- & -- & -- & -- \\
\hline \multicolumn{2}{|c|}{ Prevalencia anual de ED } & $31,3(2,5)$ & $42,6(3,2)$ & $1,62(1,14-2,30)$ & 0,07 & $1,51(1,04-2,17)$ & 0,027 \\
\hline \multicolumn{2}{|c|}{$\begin{array}{l}\text { Severidad del episodio } \\
\text { depresivo (ED) }\end{array}$} & $\begin{array}{l}\text { No } \\
\mathrm{n}=517 \\
\%(\mathrm{ES})\end{array}$ & $\begin{array}{c}\text { Sí } \\
\mathrm{n}=325 \\
\%(\mathrm{ES})\end{array}$ & $\begin{array}{c}\text { OR } \\
(\mathrm{IC} 95 \%)\end{array}$ & $\mathrm{P}$ & OR ajustado (IC95\%) & $\mathrm{p}$ \\
\hline \multirow{3}{*}{$\begin{array}{l}\text { Prevalencia } \\
\text { de vida de } \\
\text { ED }\end{array}$} & Leve & $23,8(2,2)$ & $4,6(1,3)$ & 1(Referencia) & \multirow{3}{*}{$<0,001$} & 1(Referencia) & \multirow{3}{*}{$<0,001$} \\
\hline & Moderado & $36,1(2,5)$ & $24,8(2,9)$ & $3,53(1,78-6,98)$ & & $3,76(1,89-7,51)$ & \\
\hline & Severo & $40,0(2,6)$ & $70,5(3,1)$ & $9,05(4,77-17,16)$ & & $9,67(5,05-18,50)$ & \\
\hline \multirow{4}{*}{$\begin{array}{l}\text { Prevalencia } \\
\text { anual de ED }\end{array}$} & No & $68,7(2,5)$ & $57,4(3,2)$ & 1(Referencia) & \multirow{4}{*}{$<0,001$} & 1(Referencia) & \multirow{4}{*}{$<0,001$} \\
\hline & Leve & $7,5(1,4)$ & $1,7(0,8)$ & $0,26(0,09-0,77)$ & & $0,21(0,07-0,65)$ & \\
\hline & Moderado & $11,3(1,7)$ & $9,9(2,1)$ & $1,04(0,58-1,87)$ & & $0,97(0,52-1,83)$ & \\
\hline & Severo & $12,5(1,8)$ & $31,0(3,2)$ & $2,95(1,91-4,57)$ & & $2,88(1,87-4,44)$ & \\
\hline
\end{tabular}

${ }^{a}$ Episodio depresivo de mayor severidad en la vida definido como el periodo más severo de dos semanas o más en las que se sentía deprimido(a) sin interés por la mayor parte de las cosas o cansado(a) casi todos los días

${ }^{\mathrm{b}}$ Asociación cruda (Regresión logística simple para muestras complejas/Análisis bivariado para variables categóricas; en el caso de medias se utilizó el modelo lineal general para muestras complejas

${ }^{\mathrm{C}}$ Controlado por variables sociodemográficas (sexo, edad, estado civil, estado laboral, grado de instrucción y nivel de pobreza) modelado con regresión logística para muestras complejas

fueron similares, sin embargo, controlando con las demás variables sociodemográficas, se identificó una asociación sigtablnificativa $(\mathrm{p}=0,049)$, con una probabilidad de 1,477 mayor en las personas desocupadas.

Edad de inicio del ED con y sin irritabilidad y asociación entre la presencia de IED y severidad del $\mathrm{ED}$

Se encontró una edad promedio menor de inicio del primer ED estadísticamente significativa en las personas con ED que presentaron irritabilidad (27,8 años), en comparación con las personas que no presentaron irritabilidad en ED (32,3 años), aun controlando con variables sociodemográficas (tabla 2).

En cuanto a la prevalencia de vida del ED según nivel de severidad, se encontró que el $70,5 \%$ de adultos presentó irritabilidad dentro de un ED severo, con un OR de 9,051, lo que indica que aquellos adultos con ED severo tienen 9 veces más probabilidad de presentar irritabilidad, en comparación del ED leve, y 3 veces más probabilidad, en comparación del
ED moderado. Tanto en el análisis bivariado como en el análisis multivariado controlado con factores sociodemográficos, esta variable de asociación es altamente significativa $(\mathrm{p}<0,001)$, lo que implica que existe una asociación directa entre la severidad del ED y la presencia de irritabilidad en ED. En cuanto a la prevalencia anual de ED según severidad, también existió mayor prevalencia de irritabilidad en adultos con ED severo $(31,1 \%)$, seguida del ED moderado $(9,9 \%)$, y luego del ED leve $(1,7 \%)$. Los valores de OR del ED leve anual y ED severo anual fueron de 0,266 y 2,958 respectivamente, lo que indica que las personas con ED severo anual presentaron casi 3 veces mayor probabilidad de presentar irritabilidad que las que presentan ED leve; siendo todas estas asociaciones altamente significativas $(\mathrm{p}<0,001)$.

\section{Asociación entre la presencia de IED y comorbilidad con trastornos mentales}

La prevalencia de vida y anual de cualquier trastorno de ansiedad y de trastorno de ansiedad generalizada (TAG) pura presentaron asociación significativa, tanto en el análisis bivariado como multivariado. Asimismo, se encontró que la prevalencia de vida de abuso o 
Salazar-Saavedra Y, Saavedra-Castillo J.

Tabla 3. Asociación entre presencia de irritabilidad y comorbilidad con trastornos mentales en adultos con ED (CIE-10) ${ }^{\mathrm{a}}$

\begin{tabular}{|c|c|c|c|c|c|c|}
\hline \multirow[b]{2}{*}{ Variable } & \multicolumn{2}{|c|}{$\begin{array}{l}\text { Síntomas de } \\
\text { irritabilidad }\end{array}$} & \multicolumn{2}{|c|}{ Modelo $1^{\text {b }}$} & \multicolumn{2}{|c|}{ Modelo $2^{\mathrm{c}}$} \\
\hline & $\begin{array}{c}\text { No } \\
\mathbf{n}=\mathbf{5 1 7} \\
\%(\mathbf{E S})\end{array}$ & $\begin{array}{c}\text { Sí } \\
\mathrm{N}=325 \\
\%(\mathbf{E S})\end{array}$ & $\begin{array}{c}\text { OR } \\
(\text { IC 95\%) }\end{array}$ & $\mathbf{P}$ & $\begin{array}{l}\text { OR ajustado } \\
\text { (IC95\%) }\end{array}$ & $\mathbf{p}$ \\
\hline $\begin{array}{l}\text { Prevalencia de vida de cualquier } \\
\text { trastorno de ansiedad (TAG* pura } \\
\text { con y sin depresión) }\end{array}$ & $25,0(2,3)$ & $39,6(3,6)$ & $1,96(1,33-2,88)$ & 0,001 & $2,00(1,35-2,98)$ & 0,001 \\
\hline $\begin{array}{l}\text { Prevalencia anual de cualquier } \\
\text { trastorno de ansiedad (TAG pura } \\
\text { con o sin depresión) }\end{array}$ & $4,7(1,1)$ & $9,2(1,8)$ & $3,88(2,26-6,66)$ & $<0,001$ & $3,65(2,07-6,44)$ & $<0,001$ \\
\hline $\begin{array}{l}\text { Prevalencia de vida de TAG pura } \\
\text { (con o sin depresión) }\end{array}$ & $8,5(1,5)$ & $23,0(3,1)$ & $3,19(1,95-5,32)$ & $<0,001$ & $3,17(1,84-5,45)$ & $<0,001$ \\
\hline $\begin{array}{l}\text { Prevalencia anual de TAG pura } \\
\text { (con o sin depresión) }\end{array}$ & $2,6(0,7)$ & $13,8(2,8)$ & $5,97(2,92-12,20)$ & $<0,001$ & $5,93(2,71-12,97)$ & $<0,001$ \\
\hline Prevalencia de vida de fobia social & $4,1(1,3)$ & $4,7(1,2)$ & $1,15(0,50-2,67)$ & 0,731 & $1,17(0,46-2,96)$ & 0,733 \\
\hline Prevalencia de vida de TEPT** & $14,6(1,9)$ & $14,5(2,3)$ & $0,99(0,62-1,59)$ & 0,982 & $1,05(0,63-1,74)$ & 0,844 \\
\hline $\begin{array}{l}\text { Prevalencia de vida de abuso/ } \\
\text { dependencia de alcohol (criterios } \\
\text { MINI***) }\end{array}$ & $9,5(1,6)$ & $20,8(3,1)$ & $2,52(1,50-4,21)$ & $<0,001$ & $2,30(1,26-4,19)$ & 0,006 \\
\hline $\begin{array}{l}\text { Prevalencia anual de abuso/ } \\
\text { dependencia de alcohol (criterios } \\
\text { MINI) }\end{array}$ & $2,8(0,8)$ & $5,0(1,6)$ & $1,78(0,78-4,08)$ & 0,170 & $1,35(0,54-3,35)$ & 0,511 \\
\hline $\begin{array}{l}\text { Prevalencia anual de abuso/ } \\
\text { dependencia a tabaco }\end{array}$ & $1,2(0,5)$ & $2,3(1,1)$ & $1,91(0,56-6,50)$ & 0,297 & $1,72(0,48-6,12)$ & 0,397 \\
\hline
\end{tabular}

dependencia de alcohol se asocia directamente a la presencia de IED, aun controlando con factores sociodemográficos (tabla 3).

No se halló significancia estadística en cuanto a la prevalencia de vida de fobia social, o de trastorno de estrés post traumático, ni a la prevalencia anual de abuso/dependencia de alcohol o de tabaco.

\section{Asociación entre la presencia de IED y síntomas depresivos}

En cuanto a la asociación de los síntomas depresivos con la presencia de irritabilidad en ED, se encontró en los análisis multivariados controlando con factores sociodemográficos y con la severidad el ED, que los síntomas que tuvieron asociación significativa con la variable de estudio fueron los siguientes: autorreproches, problemas de concentración, y pensamientos suicidas. Mientras que aquellos que solo presentaron asociación significativa aun siendo controlados por factores sociodemográficos fueron la falta de energía, agitación o inhibición psicomotriz y sentimientos de inferioridad. Otros síntomas que mostraron una tendencia asociativa fueron: anhedonia $(p=0,052)$, problemas de sueño $(p=0,069)$ y agitación o disminución psicomotriz $(\mathrm{p}=0,081)$ (tabla 4$)$.

\section{Asociación entre la presencia de IED y conductas suicidas-conductas violentas}

Las personas con IED presentaron una prevalencia significativamente mayor de deseos de morir en la vida $(62,1 \%$ ES 3,3) y en el último año (19,4\% ES $2,9)$ en comparación con las personas deprimidas sin irritabilidad, aunque no se encontró asociación estadísticamente significativa al controlarse con factores sociodemográficos y severidad de ED. En el caso de pensamiento e intento suicida las prevalencias de vida y anuales son más elevadas en las personas con IED (39,9\% y 13,3\% respectivamente) en comparación que los deprimidos sin irritabilidad (18,9\% y 5,2\% 
Factores asociados a irritabilidad en adultos con episodio depresivo en Lima Metropolitana.

Tabla 4. Asociación entre presencia de irritabilidad y síntomas depresivos en adultos con ED (CIE-10)a

\begin{tabular}{|c|c|c|c|c|c|}
\hline \multirow{3}{*}{ Síntomas depresivos CIE-10/DSM-IV } & \multicolumn{2}{|c|}{$\begin{array}{l}\text { Síntoma de } \\
\text { irritabilidad }\end{array}$} & \multirow[b]{3}{*}{ Modelo $1^{\text {b }}$} & \multirow[t]{2}{*}{ Valor de p } & \multirow[b]{3}{*}{ Modelo $3^{\mathrm{d}}$} \\
\hline & No & Sí & & & \\
\hline & $\begin{array}{l}n=517 \\
\%(E S)\end{array}$ & $\begin{array}{l}\mathrm{N}=325 \\
\%(\mathrm{ES})\end{array}$ & & Modelo $2^{\mathrm{c}}$ & \\
\hline $\begin{array}{l}\text { ¿Se ha sentido especialmente triste, decaído(a), } \\
\text { deprimido(a) la mayor parte del tiempo a lo largo del día, y } \\
\text { así, casi todos los días?* }\end{array}$ & $98,6(0,5)$ & $97,4(1,0)$ & 0,255 & 0,322 & 0,205 \\
\hline $\begin{array}{l}\text { ¿Tenía casi todo el tiempo el sentimiento de no tener ganas } \\
\text { de nada, de haber perdido el interés o el placer por las cosas } \\
\text { que le agradan habitualmente?* }\end{array}$ & $95,6(1,0)$ & $94,6(1,4)$ & 0,577 & 0,734 & 0,052 \\
\hline ¿Se sentía casi todo el tiempo cansado(a), sin energía?* & $80,8(2,1)$ & $92,6(1,9)$ & $<0,001$ & $<0,001$ & 0,114 \\
\hline $\begin{array}{l}\text { ¿Su apetito había cambiado notablemente? Y ¿había } \\
\text { aumentado o perdido peso sin tener esa intención?* }\end{array}$ & $87,1(1,6)$ & $86,7(2,4)$ & 0,886 & 0,964 & 0,004 \\
\hline $\begin{array}{l}\text { ¿Tenía problemas de sueño casi todas las noches como: } \\
\text { demorarse en dormir, despertarse durante la noche o se le } \\
\text { iba el sueño muy temprano, ¿o su sueño estaba aumentado } \\
\text { (hipersomnia)?* }\end{array}$ & $82,5(1,9)$ & $86,4(2,4)$ & 0,227 & 0,121 & 0,069 \\
\hline $\begin{array}{l}\text { ¿Hablaba o se movía más lentamente de lo habitual, o al } \\
\text { contrario se sentía más agitado(a) y tenía dificultad para } \\
\text { permanecer quieto(a)?* }\end{array}$ & $58,8(2,5)$ & $67,8(3,3)$ & 0,028 & 0,015 & 0,081 \\
\hline $\begin{array}{l}\text { ¿Le faltaba la confianza en usted mismo(a), o se sentía sin } \\
\text { valor, inferior a los demás?** }\end{array}$ & $61,2(2,5)$ & $81,7(2,6)$ & $<0,001$ & $<0,001$ & 0,925 \\
\hline ¿Se hacía reproches, o se sentía culpable?* & $53,3(2,7)$ & $80,4(2,8)$ & $<0,001$ & $<0,001$ & 0,020 \\
\hline $\begin{array}{l}\text { ¿Tenía dificultad para reflexionar o para concentrarse, o } \\
\text { para tomar decisiones?* }\end{array}$ & $50,7(2,8)$ & $80,6(2,7)$ & $<0,001$ & $<0,001$ & 0,001 \\
\hline $\begin{array}{l}\text { ¿Tenía varias veces ideas negativas como pensar que } \\
\text { sería mejor que estuviese muerto(a), o pensaba en hacerse } \\
\text { daño?* }\end{array}$ & $27,6(2,3)$ & $59,8(3,4)$ & $<0,001$ & $<0,001$ & $<0,001$ \\
\hline $\begin{array}{l}\text { *Criterios diagnósticos del CIE-10 y DSM-IV. **Criterio } \\
\text { perteneciente solo al CIE-10 } \\
\text { a Episodio depresivo de mayor severidad en la vida definido como } \\
\text { el periodo más severo de dos semanas o más en las que se sentía } \\
\text { deprimido(a) sin interés por la mayor parte de las cosas o cansado(a) } \\
\text { casi todos los días }\end{array}$ & $\begin{array}{l}{ }^{\mathrm{c}} \text { Controlado } \\
\text { estado labo } \\
\text { regresión lo }\end{array}$ & $\begin{array}{l}\text { or variables } \\
\text { 1, grado de in } \\
\text { stica para mu }\end{array}$ & $\begin{array}{l}\text { ociodemográfi } \\
\text { trucción y nive } \\
\text { tras complejas }\end{array}$ & $\begin{array}{l}\text { (sexo, edac } \\
\text { l de pobreza) }\end{array}$ & $\begin{array}{l}\text { estado civil, } \\
\text { modelado con }\end{array}$ \\
\hline $\begin{array}{l}\text { b Asociación cruda (regresión logística simple para muestras } \\
\text { complejas) }\end{array}$ & $\begin{array}{l}\text { ¿ Controlad } \\
\text { estado labo } \\
\text { la depresión } \\
\text { para muestr }\end{array}$ & $\begin{array}{l}\text {, grado de i } \\
\text { leve, modera } \\
\text { complejas }\end{array}$ & $\begin{array}{l}\text { iodemográfi } \\
\text { sevión y nive }\end{array}$ & $\begin{array}{l}\text { las (sexo, edac } \\
1 \text { de pobreza) } \\
\text { lelado con regr }\end{array}$ & $\begin{array}{l}\text { estado civil, } \\
\text { severidad de } \\
\text { esión logística }\end{array}$ \\
\hline
\end{tabular}

respectivamente), y la asociación se mantiene estadísticamente significativas aun controlando con factores sociodemográficos y severidad de la depresión, excepto para el intento suicida en el último año (Tabla 5).

En cuanto a las conductas violentas se demuestra que las personas deprimidas con síntomas de irritabilidad tiene 1,747 mayor probabilidad de haber presentado alguna conducta violenta (como haberle pegado a un niño menor y haberle dejado moretones, o desde los 18 años haber participado en más de una pelea que haya acabado en golpes o haber usado piedras, palos, cuchillos o armas de fuego en una pelea) alguna vez en su vida, y este factor presenta asociación significativa aun controlando con factores sociodemográficos y la severidad del ED.

\section{Asociación entre la presencia de IED y calidad de sueño.}

En general se encontró una asociación significativa entre calidad de sueño según la escala de Pittsburgh y la presencia de irritabilidad del ED. Los componentes que tuvieron asociación significativa con la presencia de IED incluso controlando con factores sociodemográficos y niveles de severidad de ED fueron la disfunción diurna y la puntuación global de calidad 
Salazar-Saavedra Y, Saavedra-Castillo J.

Tabla 5. Asociación entre presencia de irritabilidad y conductas suicidas-conductas violentas en adultos con ED (CIE-10) ${ }^{\mathrm{a}}$

\begin{tabular}{|c|c|c|c|c|c|c|c|c|}
\hline \multirow[b]{2}{*}{ Variable } & \multicolumn{2}{|c|}{$\begin{array}{l}\text { Síntomas de } \\
\text { irritabilidad }\end{array}$} & \multicolumn{2}{|c|}{ Modelo $1^{\text {b }}$} & \multicolumn{2}{|c|}{ Modelo 2 c } & \multicolumn{2}{|c|}{ Modelo $3^{\mathrm{d}}$} \\
\hline & $\begin{array}{c}\text { No } \\
\mathbf{n}=517 \\
\%(\text { ES) }\end{array}$ & $\begin{array}{c}\text { Sí } \\
\text { N=325 } \\
\% \text { (ES) }\end{array}$ & $\begin{array}{c}\text { OR } \\
\text { (IC 95\%) }\end{array}$ & $\mathbf{P}$ & OR (IC95\%) & $\mathbf{p}$ & OR ajustado & $\mathbf{P}$ \\
\hline \multicolumn{9}{|l|}{ INDICADORES SUICIDAS } \\
\hline $\begin{array}{l}\text { Deseos de morir alguna vez en } \\
\text { la vida }\end{array}$ & $45,1(2,7)$ & $62,1(3,3)$ & $1,99(1,43-2,79)$ & $<0,001$ & $1,98(1,39-2,82)$ & $<0,001$ & $1,31(0,89-1,93)$ & 0,166 \\
\hline $\begin{array}{l}\text { Deseos de morir en el último } \\
\text { año }\end{array}$ & $10,5(1,6)$ & $19,4(2,9)$ & $2,04(1,26-3,30)$ & 0,004 & $1,94(1,21-3,09)$ & 0,005 & $1,34(0,83-2,18)$ & 0,224 \\
\hline $\begin{array}{l}\text { Pensamiento suicida alguna } \\
\text { vez en la vida }\end{array}$ & $18,9(2,0)$ & $39,9(3,4)$ & $2,85(1,94-4,20)$ & $<0,001$ & $2,74(1,85-4,08)$ & $<0,001$ & $2,05(1,34-3,13)$ & 0,001 \\
\hline $\begin{array}{l}\text { Pensamientos suicidas en el } \\
\text { último año }\end{array}$ & $5,2(1,0)$ & $13,3(2,4)$ & $2,80(1,61-4,88)$ & $<0,001$ & $2,61(1,51-4,52)$ & 0,001 & $1,79(1,04-3,10)$ & 0,036 \\
\hline $\begin{array}{l}\text { Planeamiento suicida alguna } \\
\text { vez en la vida }\end{array}$ & $8,8(1,4)$ & $18,3(2,8)$ & $2,32(1,38-3,01)$ & 0,001 & $2,46(1,45-4,17)$ & 0,001 & $1,81(1,06-3,09)$ & 0,029 \\
\hline $\begin{array}{l}\text { Planeamiento suicida en el } \\
\text { último año }\end{array}$ & $2,4(0,9)$ & $4,8(1,3)$ & $2,01(0,79-5,13)$ & 0,142 & $1,85(0,74-4,60)$ & 0,183 & $1,08(0,43-2,75)$ & 0,858 \\
\hline $\begin{array}{l}\text { Intento suicida alguna vez en } \\
\text { la vida }\end{array}$ & $6,1(1,2)$ & $15,0(2,7)$ & $2,74(1,49-5,02)$ & 0,001 & $2,64(1,45-4,82)$ & 0,002 & $1,93(1,06-3,50)$ & 0,030 \\
\hline Intento suicida en el último año & $1,1(0,5)$ & $2,4(1,1)$ & $2,26(0,59-8,72)$ & 0,233 & $1,57(0,39-6,20)$ & 0,521 & $0,80(0,20-3,16)$ & 0,758 \\
\hline \multicolumn{9}{|l|}{ CONDUCTAS VIOLENTAS } \\
\hline $\begin{array}{l}\text { Prevalencia de vida de } \\
\text { tendencias violentas adultos }\end{array}$ & $27,0(2,3)$ & $41,3(3,6)$ & $1,90(1,32-2,74)$ & 0,001 & $1,66(1,11-2,47)$ & 0,012 & $1,74(1,14-2,66)$ & 0,010 \\
\hline
\end{tabular}

${ }^{a}$ Episodio depresivo de mayor severidad en la vida definido como el periodo más severo de dos semanas o más en las que se sentía deprimido(a) sin interés por la mayor parte de las cosas o cansado(a) casi todos los días

${ }^{\mathrm{b}}$ Asociación cruda (Regresión logística simple para muestras complejas /Análisis bivariado)

${ }^{\mathrm{c}}$ Controlado por variables sociodemográficas (sexo, edad, estado civil, estado laboral, grado de instrucción y nivel de pobreza) modelado con regresión logística

e Tendencias violentas definido como, si después de los 18 años, le ha pegado a un niño menor y le ha dejado moretones, o desde los 18 años ha participado en más de una pelea que haya acabado en golpes o ha usado piedras, palos, cuchillos o armas de fuego en una pelea.

\begin{abstract}
${ }^{\mathrm{d}}$ Controlado por variables sociodemográficas (sexo, edad, estado civil, estado laboral, grado de instrucción y nivel de pobreza) y severidad de la depresión (leve, moderada y severa) modelado con regresión logística para muestras complejas
\end{abstract}

de sueño de Pittsburgh. Por otro lado, la latencia de sueño presentaría una asociación significativa con la presencia de IED, incluso siendo controlada por factores sociodemográficos, pero pierde su significancia al ser controlada por la severidad del ED. Los componentes de eficiencia y perturbaciones del sueño presentaron una asociación indirecta con IED a través de los controles con factores sociodemográficos y la severidad del ED (tabla 6).

\section{Asociación entre la presencia de IED, estilos de crianza y eventos de vida adversos antes de los 18 años.}

En general se encontró una mayor prevalencia de IED con relación a varios estilos de crianza, en comparación de aquellos que no presentaron irritabilidad. La sobreprotección, favoritismo, control sin afecto, engreimiento, ansiedad de los padres a que algo malo podía suceder, y la prohibición por temor, son los que presentaron asociación significativa de manera directa con la presencia de IED, aun estando controladas por factores sociodemográficos y severidad del ED. De la misma manera, se encontró una mayor prevalencia de IED con relación a varios eventos adversos antes de los 18 años, presentando significancia estadística aun controlando con factores sociodemográficos y severidad de la depresión: alguno de los padres que llegaba ebrios a casa; padres mucho tiempo ausentes en casa; presenciar discusiones constantes entre los padres; infidelidad de alguno de sus padres; y ser víctima de abuso sexual o emocional o psicológico (tabla 7 y tabla 8 ). 
Factores asociados a irritabilidad en adultos con episodio depresivo en Lima Metropolitana.

Tabla 6. Asociación entre presencia de irritabilidad y calidad de sueño en adultos con ED (CIE-10) ${ }^{\mathrm{a}}$

\begin{tabular}{|c|c|c|c|c|c|}
\hline \multirow{3}{*}{ Variable } & \multicolumn{2}{|c|}{ Síntoma de irritabilidad } & \multirow{2}{*}{\multicolumn{3}{|c|}{ P valor }} \\
\hline & No & Sí & & & \\
\hline & $\begin{array}{l}\mathrm{n}=517 \\
\%(\mathrm{ES})\end{array}$ & $\begin{array}{l}n=325 \\
\%(E S)\end{array}$ & Modelo $1^{\mathrm{b}}$ & Modelo $2^{\mathrm{c}}$ & Modelo 3 \\
\hline \multicolumn{6}{|c|}{ En las últimas 4 semanas ¿Cómo valoraría o calificaría la calidad de sueño? } \\
\hline Bastante bueno & $6,5(1,2)$ & $8,3(2,1)$ & \multirow{4}{*}{0,059} & \multirow{4}{*}{0,046} & \multirow{4}{*}{0,324} \\
\hline Bueno & $74,2(2,4)$ & $63,6(3,1)$ & & & \\
\hline Malo & $18,1(2,1)$ & $27,0(2,9)$ & & & \\
\hline Bastante malo & $1,2(0,6)$ & $1,0(0,5)$ & & & \\
\hline \multicolumn{3}{|l|}{ Latencia del sueño } & \multirow{5}{*}{0,024} & \multirow{5}{*}{0,008} & \multirow{5}{*}{0,062} \\
\hline Apropiada & $42,5(2,8)$ & $35,5(3,2)$ & & & \\
\hline Leve alteración & $36,9(2,6)$ & $32,5(3,2)$ & & & \\
\hline Moderada alteración & $14,0(1,7)$ & $24,2(3,2)$ & & & \\
\hline Severa alteración & $6,6(1,4)$ & $7,8(1,9)$ & & & \\
\hline \multicolumn{3}{|l|}{ Duración del sueño } & \multirow{5}{*}{0,990} & \multirow{5}{*}{0,944} & \multirow{5}{*}{0,955} \\
\hline Más de $7 \mathrm{~h}$ & $38,0(2,4)$ & $38,9(3,3)$ & & & \\
\hline Entre 6 y $7 \mathrm{~h}$ & $29,4(2,3)$ & $28,7(2,9)$ & & & \\
\hline Entre 5 y $6 h$ & $27,4(2,3)$ & $26,8(3,0)$ & & & \\
\hline Menos de 5 horas & $5,2(1,0)$ & $5,5(1,4)$ & & & \\
\hline \multicolumn{3}{|l|}{ Eficiencia del sueño } & \multirow{5}{*}{0,278} & \multirow{5}{*}{0,131} & \multirow{5}{*}{0,036} \\
\hline Más del 85\% & $76,7(2,4)$ & $76,7(3,0)$ & & & \\
\hline Del 75 al 84\% & $15,9(2,1)$ & $12,8(2,2)$ & & & \\
\hline Del 65 al $74 \%$ & $4,7(1,2)$ & $4,2(1,3)$ & & & \\
\hline Menos del 65\% & $2,7(0,9)$ & $6,3(2,1)$ & & & \\
\hline \multicolumn{3}{|c|}{ Perturbaciones del sueño en el último mes } & \multirow{4}{*}{0,051} & \multirow{4}{*}{0,027} & \multirow{4}{*}{0,029} \\
\hline Ninguna perturbación & $38,7(2,7)$ & $28,5(3,3)$ & & & \\
\hline Entre 1 y 9 & $56,2(2,7)$ & $64,9(3,4)$ & & & \\
\hline Entre 10 y 18 & $5,1(1,3)$ & $6,6(1,4)$ & & & \\
\hline
\end{tabular}

¿Cuántas veces habrá tomado medicinas para dormir por su cuenta o recetadas por el médico?

\begin{tabular}{|c|c|c|c|c|c|}
\hline Ninguna vez en las últimas 4 semanas & $88,3(2,0)$ & $85,4(2,4)$ & \multirow{4}{*}{0,772} & \multirow{4}{*}{0,734} & \multirow{4}{*}{0,971} \\
\hline Menos de una vez a la semana & $3,1(1,0)$ & $3,4(1,0)$ & & & \\
\hline Una o dos veces a la semana & $3,5(1,3)$ & $3,8(1,1)$ & & & \\
\hline Tres o más veces a la semana & $5,1(1,2)$ & $7,4(2,1)$ & & & \\
\hline \multicolumn{6}{|l|}{ Disfunción diurna } \\
\hline Ninguna & $60,6(2,7)$ & $43,8(3,4)$ & \multirow{4}{*}{$<0,001$} & \multirow{4}{*}{$<0,001$} & \multirow{4}{*}{0,008} \\
\hline Leve & $33,3(2,6)$ & $41,3(3,2)$ & & & \\
\hline Moderada & $4,8(1,2)$ & $13,0(2,5)$ & & & \\
\hline Severa & $1,4(0,7)$ & $2,0(0,8)$ & & & \\
\hline \multicolumn{6}{|l|}{ CSP Puntuación global (Categórico) } \\
\hline in problema de sueño & $55,1(2,7)$ & $40,8(3,4)$ & \multirow{4}{*}{0,001} & \multirow{4}{*}{$<0,001$} & \multirow{4}{*}{0,008} \\
\hline Merece atención médica & $31,4(2,6)$ & $34,7(3,2)$ & & & \\
\hline Ierece atención y tratamiento médico & $12,9(1,8)$ & $24,4(3,1)$ & & & \\
\hline trata de un problema de sueño grave & $0,6(0,4)$ & $0,1(0,1)$ & & & \\
\hline \multicolumn{2}{|c|}{$\begin{array}{l}\text { Episodio depresivo de mayor severidad en la vida definido } \\
\text { como el periodo más severo de dos semanas o más en las } \\
\text { que se sentía deprimido(a) sin interés por la mayor parte de } \\
\text { as cosas o cansado(a) casi todos los días }\end{array}$} & \multicolumn{4}{|c|}{$\begin{array}{l}{ }^{\mathrm{c}} \text { Controlado por variables sociodemográficas (sexo, edad, } \\
\text { estado civil, estado laboral, grado de instrucción y nivel de } \\
\text { pobreza) modelado con regresión logística }\end{array}$} \\
\hline \multicolumn{2}{|c|}{$\begin{array}{l}\text { Asociación cruda (Regresión logística simple para muestras } \\
\text { complejas /Análisis bivariado) }\end{array}$} & \multicolumn{4}{|c|}{$\begin{array}{l}\text { d Controlado por variables sociodemográficas (sexo, edad, } \\
\text { estado civil, estado laboral, grado de instrucción y nivel } \\
\text { de pobreza) y severidad de la depresión (leve, moderada } \\
\text { y severa) modelado con regresión logística para muestras } \\
\text { complejas }\end{array}$} \\
\hline
\end{tabular}


Salazar-Saavedra Y, Saavedra-Castillo J.

Tabla 7.- Asociación entre presencia de irritabilidad y estilos de crianza en adultos con ED (CIE-10) ${ }^{\mathrm{a}}$

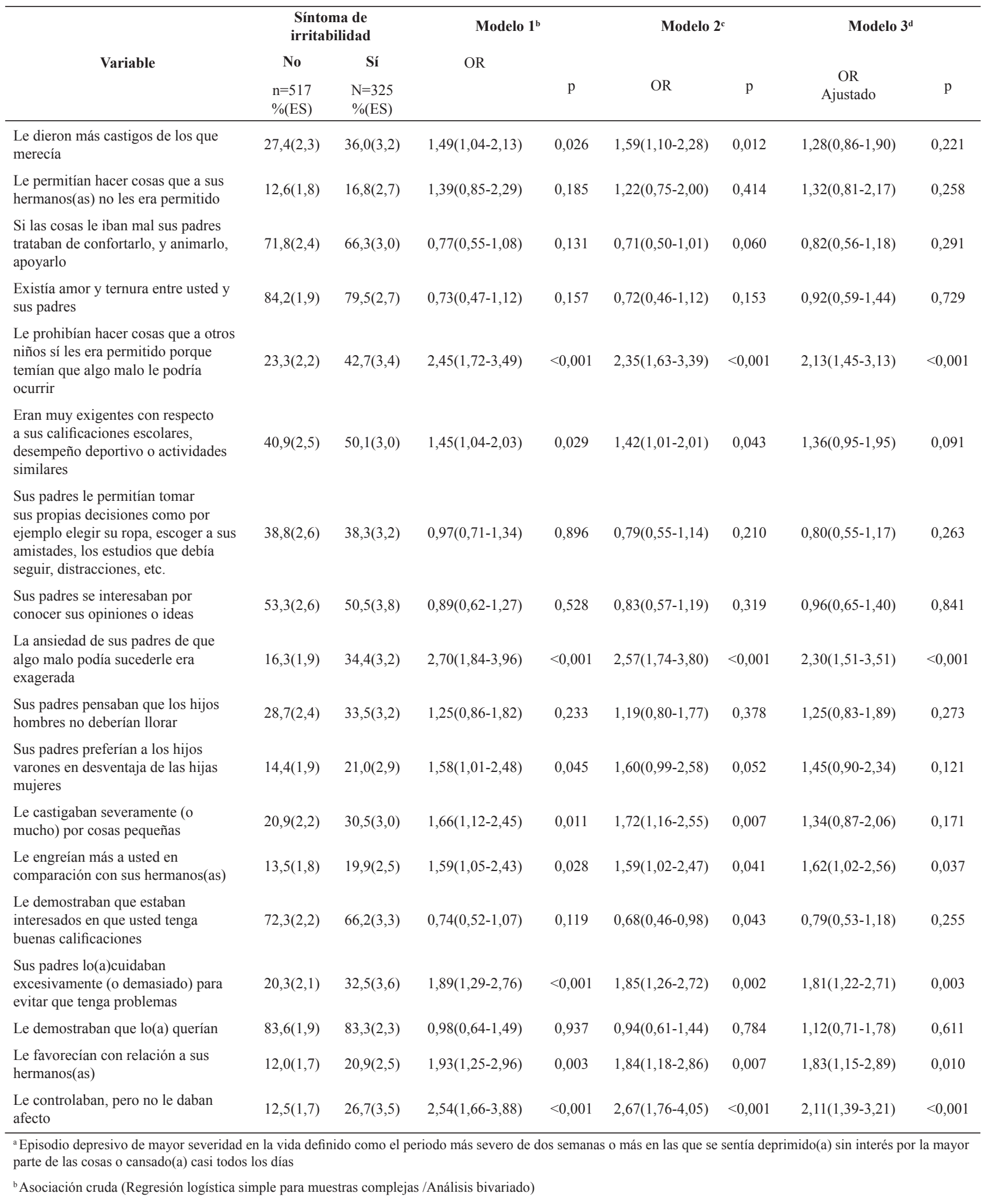


Factores asociados a irritabilidad en adultos con episodio depresivo en Lima Metropolitana.

Tabla 8. Asociación entre irritabilidad y eventos adversos antes de los 18 años en adultos con ED (CIE-10)a

\begin{tabular}{|c|c|c|c|c|c|c|c|c|}
\hline \multirow{3}{*}{ Variable } & \multicolumn{2}{|c|}{$\begin{array}{c}\text { Síntoma de } \\
\text { irritabilidad }\end{array}$} & & \multicolumn{2}{|c|}{ Modelo $2^{\mathrm{c}}$} & \multicolumn{2}{|c|}{ Modelo $3^{\mathrm{d}}$} \\
\hline & No & Sí & OR & & & & & \\
\hline & $\begin{array}{l}\mathrm{n}=517 \\
\%(\mathrm{ES})\end{array}$ & $\begin{array}{l}\mathrm{n}=325 \\
\%(\mathrm{ES})\end{array}$ & & $\mathrm{p}$ & OR & $\mathrm{p}$ & OR Ajustado & $\mathrm{p}$ \\
\hline $\begin{array}{l}\text { Cuando era menor de } 11 \text { años } \\
\text { sus padres discutían mucho }\end{array}$ & $37,3(2,6)$ & $50,6(3,7)$ & $1,72(1,20-2,45)$ & 0,003 & $1,55(1,07-2,25)$ & 0,020 & $1,41(0,96-2,07)$ & 0,080 \\
\hline $\begin{array}{l}\text { Alguno de sus padres llegaba } \\
\text { ebrio o borracho a casa con } \\
\text { regularidad (o frecuencia) }\end{array}$ & $32,2(2,5)$ & $43,8(3,5)$ & $1,64(1,15-2,32)$ & 0,005 & $1,54(1,06-2,25)$ & 0,023 & $1,50(1,02-2,23)$ & 0,039 \\
\hline $\begin{array}{l}\text { Alguno de sus padres estaba } \\
\text { mucho tiempo ausente en casa }\end{array}$ & $32,4(2,5)$ & $44,5(3,7)$ & $1,66(1,15-2,40)$ & 0,006 & $1,61(1,11-2,35)$ & 0,012 & $1,53(1,03-2,27)$ & 0,032 \\
\hline $\begin{array}{l}\text { Alguno de sus padres fue infiel } \\
\text { en la relación }\end{array}$ & $22,6(2,3)$ & $35,7(3,3)$ & $1,90(1,30-2,77)$ & $<0,001$ & $1,88(1,27-2,79)$ & 0,002 & $1,90(1,25.2,87)$ & 0,003 \\
\hline $\begin{array}{l}\text { Alguno de sus padres lo(a) } \\
\text { amenazaba con no quererlo(a) }\end{array}$ & $4,1(1,0)$ & $9,7(2,1)$ & $2,49(1,26-4,94)$ & 0,009 & $2,36(1,23-4,53)$ & 0,010 & $1,84(0,96-3,54)$ & 0,066 \\
\hline $\begin{array}{l}\text { Alguno de sus padres lo(a) } \\
\text { amenazaba con matarlo(a) }\end{array}$ & $1,5(0,7)$ & $2,9(1,1)$ & $2,01(0,56-7,18)$ & 0,282 & $1,82(0,54-6,05)$ & 0,328 & $1,47(0,42-5,07)$ & 0,540 \\
\hline $\begin{array}{l}\text { Alguno de sus padres lo(a) hacía } \\
\text { sentir culpable por desacuerdos } \\
\text { o discusiones de la familia }\end{array}$ & $5,9(1,2)$ & $11,3(2,1)$ & $2,03(1,12-3,66)$ & 0,019 & $1,92(1,07-3,43)$ & 0,027 & $1,62(0,89-2,94)$ & 0,110 \\
\hline $\begin{array}{l}\text { Alguno de sus padres lo(a) } \\
\text { amenazaba con abandonarlo }\end{array}$ & $3,4(1,0)$ & $7,6(1,8)$ & $2,36(1,07-5,18)$ & 0,033 & $2,15(1,01-4,56)$ & 0,045 & $1,50(0,71-3,18)$ & 0,280 \\
\hline $\begin{array}{l}\text { Sus padres se separaron o } \\
\text { divorciaron }\end{array}$ & $25,1(2,1)$ & $31,5(3,0)$ & $1,37(0,97-1,94)$ & 0,074 & $1,34(0,94-1,91)$ & 0,106 & $1,20(0,82-1,75)$ & 0,330 \\
\hline $\begin{array}{l}\text { Mientras crecía sufrió algún tipo } \\
\text { de abuso físico como golpes, } \\
\text { puñetes o empujones en forma } \\
\text { frecuente }\end{array}$ & $19,3(2,1)$ & $28,4(3,0)$ & $1,65(1,10-2,47)$ & 0,015 & $1,64(1,11-2,43)$ & 0,012 & $1,22(0,82-1,81)$ & 0,320 \\
\hline $\begin{array}{l}\text { Mientras crecía sufrió algún tipo } \\
\text { de abuso sexual como ser objeto } \\
\text { (o haber sufrido) de intentos o } \\
\text { actos sexuales inapropiados, } \\
\text { forzados en contra de su } \\
\text { voluntad o bajo amenazas }\end{array}$ & $8,7(1,3)$ & $18,3(2,5)$ & $2,33(1,43-3,79)$ & 0,001 & $2,47(1,51-4,03)$ & $<0,001$ & $1,90(1,14-3,16)$ & 0,013 \\
\hline $\begin{array}{l}\text { Mientras crecía, su familia } \\
\text { vivió en permanente riesgo o } \\
\text { amenaza a la integridad física de } \\
\text { alguno de sus miembros }\end{array}$ & $5,3(1,3)$ & $6,3(1,5)$ & $1,19(0,59-2,37)$ & 0,621 & $0,99(0,47-2,07)$ & 0,989 & $0,73(0,33-1,59)$ & 0,432 \\
\hline $\begin{array}{l}\text { Mientras crecía recibió insultos, } \\
\text { agresiones verbales u ofensas en } \\
\text { forma frecuente }\end{array}$ & $21,8(2,1)$ & $36,7(3,2)$ & $2,08(1,437-3,015)$ & $<0,001$ & $2,10(1,44-3,07)$ & $<0,001$ & $1,54(1,02-2,30)$ & 0,037 \\
\hline $\begin{array}{l}\text { Mientras crecía fue objeto (o } \\
\text { haber sufrido) de chantajes, } \\
\text { manipulaciones o humillaciones } \\
\text { en forma frecuente }\end{array}$ & $9,8(1,6)$ & $24,0(2,9)$ & $2,90(1,819-4,637)$ & $<0,001$ & $2,82(1,77-4,49)$ & $<0,001$ & $2,07(1,24-3,44)$ & 0,005 \\
\hline $\begin{array}{l}\text { Mientras crecía se ha encontrado } \\
\text { en situaciones en las que se ha } \\
\text { sentido abandonado(a) porque } \\
\text { no le procuraban alimentos, } \\
\text { medicinas, ropa adecuada, no } \\
\text { lo(a) han llevado al médico } \\
\text { o no le han brindado otras } \\
\text { necesidades básicas o afecto. }\end{array}$ & $14,8(1,9)$ & $24,7(3,0)$ & $1,88(1,231-2,883)$ & 0,004 & $1,79(1,16-2,77)$ & 0,009 & $1,32(0,84-2,05)$ & 0,219 \\
\hline
\end{tabular}

${ }^{a}$ Episodio depresivo de mayor severidad en la vida definido como el periodo más severo de dos semanas o más en las que se sentía deprimido(a) sin interés por la mayor parte de las cosas o cansado(a) casi todos los días

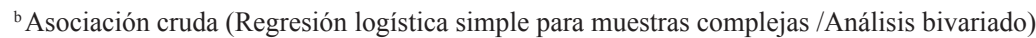




\section{DISCUSIÓN}

Es importante señalar que este estudio es el primer estudio de tipo poblacional realizado en nuestro medio y en la región, que busca resaltar la importancia del componente de irritabilidad en el ED. En líneas generales, este estudio confirma que la depresión con irritabilidad presenta múltiples aspectos que la diferencia de la depresión sin este síntoma, y que la configuraría como un problema de mayor complejidad dentro del trastorno depresivo.

El sentido de que las variables hayan sido ajustadas o controladas con factores sociodemográficos, es debido a que se ha comprobado que éstos contribuirían de distintas maneras a la manifestación de dicho síntoma, no solo dentro de un ED, sino de otros trastornos. Asimismo, se decidió incluir controlar las variables con la severidad del ED debido a que estudios anteriores demostraron que existe asociación significativa en los resultados obtenidos; además, existe la posibilidad de que la severidad del ED pudiera explicar la manifestación de algunos síntomas por lo cual se asume que está asociada la irritabilidad, pudiendo ser la severidad del ED la que se asocie directamente con la irritabilidad y no la presencia de otro factor $(13,17-$ 19).

\section{Prevalencia de IED y edad de inicio}

Nuestro estudio confirma una prevalencia significativa de la presencia de irritabilidad en adultos con ED, mayor a la prevalencia de otros estudios anteriores, como el de Fava y colaboradores (19). En cuanto a la edad de inicio de IED, en nuestro estudio fue a una edad más temprana que la media de la edad reportada en aquellos que no presentaron irritabilidad entro del ED, resultado que guarda relación con los hallazgos del estudio anteriormente mencionado.

\section{Factores sociodemográficos}

En cuanto al sexo, en nuestro estudio, se observa que los varones fueron aquellos que presentaron mayor prevalencia de IED, con una ligera tendencia asociativa $(\mathrm{p}=0,099)$; resultado que no guarda relación con los estudios revisados $(13,17,19)$, pues en éstos, las mujeres son las que tienen mayor tendencia a presentar dicho síntoma dentro del cuadro depresivo, siendo estos hallazgos estadísticamente significativos. Adicionalmente, en los hallazgos del estudio de Kovess-Masfety y colaboradores (33), no se encontró diferencia consistente entre la irritabilidad entre hombres y mujeres, pero hacen acápite a ciertas teorías socioculturales en las que consideran al síntoma de irritabilidad como un posible equivalente masculino en la depresión. Asimismo, dicho estudio encontró evidencia en la que la depresión irritable era más común en hombres jóvenes pertenecientes a ciudades de bajos y medios ingresos.

Con relación al estado civil, nuestro estudio halló asociación entre ser soltero y presentar IED, así como una mayor prevalencia, aunque esta asociación desaparece al ser controlada con los demás factores sociodemográficos. En los estudios de Fava et al $(17,19)$, sí se encuentra significancia estadística en cuanto a la asociación de ser soltero con la presencia de IED; mientras que en el estudio de Perlis et al, si bien la prevalencia de IED se observó en personas solteras, esta asociación no alcanzó significancia estadística (13), lo que este estudio posee mayor coincidencia con los resultados de nuestro estudio.

En cuanto a los resultados en los grupos etarios, nuestro estudio muestra que las personas entre los $18 \mathrm{y}$ 44 años presentan mayor prevalencia de IED, mientras que los adultos mayores de 60 años presentan menor prevalencia de este síntoma. Sin embargo, al igual que el estado civil, la variable de grupo etario también pierde su significancia estadística al ser controlada con los demás factores sociodemográficos. Estos resultados guardan relación muy estrecha con los hallazgos del estudio de Fava et al., (19), ya que los grupos etarios anteriormente mencionados, presentan la misma asociación con la presencia de IED, pero la diferencia se encuentra en que esta variable sí posee significancia estadística.

Cabe resaltar que, en cuanto a la condición laboral, la situación de persona desocupada fue el único factor que presentó significancia estadística, luego de haber sido controlada con factores sociodemográficos. Otros factores que no presentaron asociación significativa fueron el nivel educativo, analfabetismos, áreas de residencia, y nivel de pobreza según necesidades básicas insatisfechas.

\section{Niveles de severidad del ED}

En cuanto a los hallazgos de nuestro estudio sobre la asociación entre la presencia de IED y el nivel de severidad del ED, encontramos que existe una mayor prevalencia de ED severo en asociación con la presencia de IED, siendo esta asociación estadísticamente significativa. Estos resultados coinciden con lo hallado en otros estudios como los de Fava et al (19) y Perlis et 
Factores asociados a irritabilidad en adultos con episodio depresivo en Lima Metropolitana.

al (13), en los que se halla que a mayor severidad del $\mathrm{ED}$, la presencia de irritabilidad es más prevalente.

\section{Comorbilidad con trastornos mentales}

En nuestro estudio, la presencia de cualquier trastorno de ansiedad y TAG pura presentaron significancia estadística, mas no se halló significancia estadística en cuanto a la prevalencia de vida de fobia social, o de trastorno de estrés post traumático, ni a la prevalencia anual de abuso/dependencia de alcohol o de tabaco. A diferencia de nuestro estudio, en la investigación de Fava et al (19) se encontró que además de la ansiedad generalizada, el trastorno obsesivo compulsivo, el trastorno del pánico, el trastorno de estrés post traumático y la fobia social fueron comorbilidades psiquiátricas que se asociaron significativamente a la presencia de IED, probablemente por el tamaño muestral utilizado en este último estudio, que fue mayor en comparación al nuestro. Adicionalmente, en el estudio sistemático de Vidal-Rivas et al, se encontró que la irritabilidad (en ausencia de depresión), estaría asociada a la aparición futura de depresión y síntomas de ansiedad (34); esto conllevaría a plantear la idea de que la irritabilidad sería un indicador precedente de la depresión.

Nuestro estudio determina que la prevalencia de vida del abuso o dependencia de alcohol es un factor que presenta asociación significativa con la presencia de IED, aun siendo controlados con los factores sociodemográficos. Por otro lado, el estudio de Perlis et al (13) evaluaron la prevalencia de vida de enfermedades psiquiátricas y generales en personas irritables y no irritables durante el ED actual, no encontrando asociación entre la presencia de irritabilidad con la cantidad de síntomas depresivos por uso de alcohol y otras sustancias en el pasado o durante el periodo de estudio. Esta falta de asociación podría deberse a que, en el estudio de Perlis et al, esta comorbilidad psiquiátrica fue controlada solo con tres factores: edad de inicio del ED, el sexo, la severidad de la depresión; a diferencia de nuestro estudio, que fue controlada con una mayor cantidad de factores.

\section{Síntomas depresivos}

Nuestro estudio halló asociación significativa entre la presencia de IEDy los siguientes síntomas depresivos: autorreproches, problemas de concentración y los pensamientos suicidas. Esto concuerda en cierta medida con el estudio de Fava et al (19) donde se encontró que los síntomas de fatiga, autorreproche y pensamientos de muerte fueron los más significativos, planteando que estos síntomas proporcionan una diferenciación genuina entre la depresión irritable y no irritable. Mientras que Perlis et al., determinaron que el insomnio, la pérdida de peso y la disminución de la energía estuvieron asociados a la presencia de depresión irritable, más no se da una explicación sobre esta tendencia (13). Estas diferencias entre los síntomas podrían deberse al tipo de población al que fue dirigido las encuestas: Fava et al trabajaron con participantes de distintos hogares independientemente que tuvieran o no depresión; por otro lado, Perlis et al trabajaron con pacientes de consulta externa de distintas especialidades, lo que podría suponerse que el paciente estuviese llevando algún tratamiento que pueda influir en la manifestación de algunos síntomas.

\section{Conductas suicidas y conductas violentas}

En cuanto a las conductas suicidas, los pensamientos suicidas alguna vez en la vida o en el último año, los planteamientos, e intentos suicidas alguna vez en la vida, presentaron asociación significativa en nuestro estudio. Comparablemente, en el estudio de Fava et al se encontró que el intento suicida estuvo asociado a la presencia de irritabilidad, aun siendo controladas con variables como la edad, sexo y severidad del ED (17). Entre otros estudios sobre la conducta suicida, el estudio de Nock et al., determinaron que solo algunos trastornos caracterizados por la ansiedad y el pobre control de impulsos serían predictores de conductas suicidas (35).

Con relación a los hallazgos de conductas violentas (haberle pegado a un niño menor y haberle dejado moretones, o desde los 18 años haber participado en más de una pelea que haya acabado en golpes o haber usado piedras, palos, cuchillos o armas de fuego en una pelea), estas presentaron significancia estadística en nuestro estudio. En un estudio de Caprara et al., en el que investigaron la asociación entre irritabilidad y conductas violentas en adolescentes desde los 12 años hasta cumplir los 20 años, concluyó que la presencia de irritabilidad estuvo correlacionada a conductas violentas posteriores, como las agresiones físicas y verbales, así como otros episodios violentos, atribuyendo estos comportamientos a las experiencias sociales vividas por los mismos participantes, causando inestabilidad emocional (36). Por ello, es importante la evaluación de la regulación emocional, ya que juega un rol trascendental en el nexo entre la irritabilidad y las conductas violentas. 


\section{Calidad de sueño}

En nuestro estudio se encuentra, en general, una menor calidad de sueño en los adultos con IED en comparación con aquellos sin este síntoma. Específicamente se encontró asociación significativa controlando con factores sociodemográficos y severidad de la depresión con eficiencia del sueño, perturbaciones del sueño y disfunción diurna según Índice de calidad de sueño de Pittsburgh con la presencia de IED. Existen estudios sobre la asociación entre la deprivación del sueño y la presencia de irritabilidad (37). Adicionalmente, en un estudio de Franzen et al, se menciona que la relación entre una pobre calidad de sueño y la depresión sería mucho mayor si ambas se presentan a la vez, y que esta condición podría estar asociada con cualquier otro trastorno mental (38).

\section{Estilos de crianza y eventos adversos antes de los 18 años}

Los estilos de crianza que presentaron asociación con la IED en nuestro estudio fueron la sobreprotección, la ansiedad de los padres, engreimiento, cuidado excesivo, favoritismo y control sin afecto. Si bien no hay estudios que relacionen irritabilidad con estilos de crianza, el estudio de Salirrosas y Saavedra Castillo (39) determina que la calidez en la infancia, el rechazo de los padres, el control excesivo, la sobreprotección y el favoritismo relacionan la depresión con riesgo suicida, dato importante que podría ser útil para investigaciones desde otro punto de vista.

Los eventos adversos hallados en nuestro estudio fueron los siguientes: que alguno de los padres llegaba ebrio a casa, estaba muy ausente en casa, o fue infiel en la relación; ser víctima de abuso sexual; recibir agresiones verbales; y ser objeto de chantajes o manipulaciones. No se han encontrado estudios que hayan investigado la asociación entre eventos adversos e IED; sin embargo, en el EESMLMC mencionan que existirían suficientes datos para demostrar que eventos adversos como la separación de los padres, las amenazas de abandono de estos a sus niños, el control excesivo hacia el comportamiento de los niños y el descuido en la atención de los mismos provocarían disturbios emocionales en la adultez (27). Anda et al., explican que durante la niñez, estresores tales como ser víctima o testigo de violencia doméstica, pueden conllevar a una variedad de comportamientos negativos, como el abuso de sustancias, intento suicida, y trastornos depresivos (40). Además, desde el aspecto neurológico, este estudio sustenta que esta deprivación de desarrollar experiencias apropiadas en la niñez puede reducir la actividad neuronal, y como consecuencia, producir el deterioro de las estructuras $\mathrm{y}$ funciones cerebrales.

Con todo lo expuesto, y tomando en cuenta la explicación de Franzen et al., la irritabilidad podría ser parte de una forma grave, pero subsindrómica de la depresión, de una entidad psiquiátrica distinta, o de una forma de trastorno del estado de ánimo mixto (38). De manera que la irritabilidad podría llegar a ser considerado un especificador del ED. Así mismo, al estar este síntoma presente de manera relevante en distintos síndromes, podría ser considerado como un síntoma puente, es decir, un nexo importante entre los diversos trastornos mentales.

\section{Limitaciones}

En nuestro estudio existieron algunas limitaciones. En primer lugar, debido a que es un estudio transversal, no se pudo inferir relación de causalidad. Además, cabe resaltar que, para la identificación del ED, se preguntó por el peor ED de su vida, lo que pudo haber conllevado a la subjetividad en las respuestas. Otra restricción en este estudio fue que se utilizó una sola pregunta en relación con el síntoma de irritabilidad, lo que podría haber conllevado a una confiabilidad menor que a una escala compuesta por varios ítems, o podría haber introducido falsos positivos debido a la ambigüedad de algunos términos dentro de la pregunta. Sin embargo, cabe resaltar que el hecho de haber introducido el síntoma de irritabilidad dentro de las preguntas de un ED fue con el propósito de llevar a cabo estos estudios posteriores. Si bien se descartó el diagnóstico de trastorno bipolar (incluido dentro de las preguntas del MINI), no se incluyó la evaluación por un psiquiatra en cuanto a la presencia del trastorno bipolar, por lo que alguno de los participantes podría haber presentado dicho trastorno, en vez de la depresión unipolar, conllevando a sesgos de selección, y a la aparición de falsos positivos. No se consideró la duración de cada síntoma depresivo de manera individual, por lo que no se pudo asegurar si los síntomas aparecieron simultáneamente. Por otro lado, hace falta mayores estudios que puedan dilucidar otros síntomas que diferencien la depresión entre hombres y mujeres, o la presencia de comorbilidades que no sean psiquiátricas, o la existencia de diferencias interculturales.

En conclusión, coincidente con algunos estudios previos, la irritabilidad en el ED se ve asociada por 
Factores asociados a irritabilidad en adultos con episodio depresivo en Lima Metropolitana.

distintos factores, entre los cuales destacan la edad de inicio del ED, severidad del ED; trastornos de ansiedad, prevalencia de vida de abuso/dependencia de alcohol); algunos síntomas depresivos (autorreproches, problemas de concentración, y pensamientos suicidas); conductas suicidas y conductas violentas. Este estudio aporta hallazgos de factores asociados adicionales a los encontrados en literatura como una menor calidad de sueño, algunos estilos de crianza; y eventos adversos antes de los 18 años. Todo ello reforzaría la noción de un tipo de depresión con características más severas y con mayores implicancias para un plan terapéutico integral, sustentando la posibilidad de que la irritabilidad llegue a ser considerada como un especificador del ED; de esta manera, se contribuiría a desarrollar estrategias para su identificación y manejo clínico.

Declaración de conflicto de interés: Los autores declaran no tener conflictos de intereses.

\section{Correspondencia:}

Yesenia María Salazar Saavedra

Jr. Pietro Torrigiano 410, Córpac-San Borja.

Lima, Perú.

Correo electrónico: yesenia.salazar.s@upch.pe

\section{REFERENCIAS BIBLIOGRÁFICAS}

1. Cassano P, Fava M. Depression and public health: an overview. J Psychosom Res. 2002; 53(4):849-57.

2. World Health Organization. Depression. Geneva: World Health Organization; 2018. (Fecha de acceso: 15 de marzo del 2019) Disponible en: http://www. who.int/mediacentre/factsheets/fs369/en/

3. Murray CJ, Lopez AD. Alternative projections of mortality and disability by cause 1990-2020: Global Burden of Disease Study. Lancet. 1997; 349:14981504. DOI: 10.1016/S0140-6736(96)07492-2

4. Rodríguez JJ, Kohn R, Aguilar-Gaxiola S. Epidemiología de los trastornos Mentales en América Latina y el Caribe. Washington DC: Organización Panamericana de la Salud; 2009.p. 316-28.

5. Valdez W, Miranda JA. Carga de enfermedad en el Perú: Estimación de los años de vida saludables perdidos 2012. Lima: Ministerio de Salud, Dirección General de Epidemiología; 2014.p. 11-54.

6. Smith DJ, Court H, McLean G, Martin D, Langan Martin J, Guthrie B, et al. Depression and multimorbidity: a cross-sectional study of $1,751,841$ patients in primary care. J Clin Psychiatry. 2014; 75(11):1202-8. doi: 10.4088/JCP.14m09147

7. Thase ME. Managing medical comorbidities in patients with depression to improve prognosis. J Clin Psychiatry. 2016;77 (Suppl 1):22-7. doi: 10.4088/JCP.14077su1c.04

8. Kessler RC, Foster CL, Saunders WB, Stang PE. Social Consequences of Psychiatric Disorders I: Educational Attainment. Am J Psychiatry. 1995; 152:1026-132. DOI: 10.1176/ajp.152.7.1026

9. World Health Organization. The ICD-10 classification of mental and behavioral disorders: Clinical descriptions and diagnostic guidelines. Ginebra: World Health Organization; 1992.

10. Snaith RP, Taylor CM. Irritability: definition, assesment and associated factors. Br J Psychiatry. 1985; 147: 127-36.

11. Vidal-Ribas P, Brotman M, Valdivieso I, Leibenluft E, Stringaris A. The Status of Irritability in Psychiatry: A Conceptual and Quantitative Review. J Am Acad Child Adolesc Psychiatry. 2016; 55(7): 556-570. doi: 10.1016/j.jaac.2016.04.014

12. Balbuena L, Bowen R, Baetz M, Marwaha S. Mood Instability and Irritability as Core Symptoms of Major Depression: An Exploration Using Rasch Analysis Front. Psychiatry. 2016; 7(174):1-8. DOI: 10.3389/fpsyt.2016.00174

13. Perlis RH, Fraguas R, Fava M, Trivedi MH, Luther JF, Wisniewski SR et al. Prevalence and clinical correlates of irritability in mayor depressive disorder: a preliminary report from the Sequenced Treatment Alternatives to Relieve Depression study. J Clin Psychiatry. 2005; 66: 159-66.

14. Fava GA, Grandi S, Canestrari R, Molnar G. Prodromal symptoms in primary major depressive disorder. J Affect Disord. 1990; 19: 149-52.

15. Safer DJ. Irritable mood and the Diagnostic and Statistical Manual of Mental Disorders. Child and adolescent psychiatry and Mental Health. 2009; 3:35. doi: 10.1186/1753-2000-3-35.

16. Starkstein SE, Migliorelli R, Manes F, Tesón A, Petracca G, Chemerinski E, et al. The prevalence and clinical correlates of apathy and irritability in Alzheimer's disease. Eur J Neurol. 1995; 2(6):540-6. doi: 10.1111/j.1468-1331.1995.tb00171.x

17. Perlis RH, Fava M, Trivedi MH, Alpert J, Luther JF, Wisniewski SR, et al. Irritability is associated with anxiety and greater severity, but not bipolar spectrum features, in mayor depressive disorder. Acta Psychiatr Scand. 2009; 119: 282-9.

18. Judd L, Schettler P, Coryell W, Akiskal H, Fiedorowicz J. Overt Irritability/Anger in Unipolar Mayor Depressive Episodes Past and Current Characteristics and Implications for Long-term Course. JAMA psychiatry 2013; 70(11): 1171-80. doi: 10.1001/jamapsychiatry.2013.1957

19. Fava M, Hwang I, Rush AJ, Sampson N, Walters EE, Kessler RC.The importance of irritability as a symptom of major depressive disorder: results from 
the National Comorbidity Survey Replication. Molecular Psychiatry. 2010; 15: 856-67. doi: 10.1038/mp.2009.20

20. Kleinman A. Culture and Depression. NEngl J Med. 2004; 35(10):951-953.

21. Gada MT. A cross cultural study of symptomatology of depression: Eastern versus western patients. 1982; 195-202.

22. Castro-Costa E, Dewey M, Stewart R, Banerjee S, Huppert F, Mendonca-Lima C, et al. Prevalence of depressive symptoms and syndromes in later life in ten European countries. British Journal of Psychiatry. 2007; 191: 393-401.

23. Draguns Juris G., Junko Tanaka-Matsumi. Assessment of psychopathology across and within cultures: issues and findings. Behaviour Research and Terapy 2003; 41: 755-70.

24. Karasz A. Cultural differences in conceptual models of depression. Social Science and Medicine 2005; 60:1625-35.

25. Halbreich U, Alarcon RD, Calil H, Douki S, Gaszner $\mathrm{P}$, Jadresic E, et al. Culturally-sensitive complaints of depressions and anxieties in woman. Journal of Affective disorders 2007; 102: 159-76.

26. Stompe T, Ortwein Swoboda G, Chaudhry HR, Friedmann A, Wenzel T, Schanda H. Guilt and Depression: A Cross-Cultural Comparative Study. Psychopathology 2001; 34: 289-98.

27. Saavedra-Castillo J, Paz V, Robles Y, Pomalima R, Gonzales S, Zevallos S, et al. Estudio Epidemiológico de Salud Mental en Lima Metropolitana y CallaoReplicación 2012. Anales de Salud Mental.2012; 29(1):0-0. (Fecha de acceso 15 de marzo del 2019) Disponible en: http://www.insm.gob.pe/ojsinsm/ index.php/Revista1/issue/view/10

28. Saavedra-Castillo J, Arellano M, Bernal E, Chuchón V, Cutipé Y, Gonzáles S, et al. Confiabilidad y Validez de los Cuestionarios de los Estudios Epidemiológicos de Salud Mental de Lima y de la Selva Peruana. Anales de Salud Mental. 2009; 25(1):0-0. (Fecha de acceso 15 de marzo del 2019) Disponible en: http://www.insm.gob.pe/ojsinsm/index.php/ Revista1/article/view/97

29. Lecrubier Y, Weiller E, Hergueta T, Amorim P, Bonora L.I, Lépine J.P, et al. Mini International Neuropsychiatric Interview. Madrid, España: Instituto IAP; 2001.

30. Perales A, Sogi C, Sanchez E, Salas RE. Salud mental de una población urbano-marginal de Lima. Serie Monografías de Investigación $N^{\circ} 2$. Lima, Perú: Instituto Nacional de Salud Mental "Honorio Delgado-Hideyo Noguchi”; 1995.
31. Luna-Solis Y, Robles-Arana Y, Agüero-Palacios Y. Validación del índice de calidad de sueño de Pittsburgh en una muestra peruana. Anales de Salud Mental. 2015; 31: 0-0.

32. Someya T, Uehara T, Kadowaki M, Sakado K, Reist C, Tang SW, et al. Factor analysis of EMBU scale in a large sample of Japanese volunteers. Acta Psychiatr Scand. 1999; 100(4): 252-257.

33. Kovess-Masfety V, Alonso J, Angermeyer M, Bromet E, De Girolamo G, De Jonge P, et al. Irritable mood in major depressive disorder: Results from the World Mental Health Surveys. France: National Institute of Health. 2013; 30(4):395-404.

34. Vidal-Ribas P, Brotman MA, Valdivieso I, Leibeniuft E, Stringaris A. The status of irritability in Psychiatry: A conceptual and quantitive review. Journal of American Academy of Child and Adolescent Psychiatry. 2016; 1-31.

35.Nock MK, Hwang I, Sampson N, Kessler RC, Angermeyer M, Beautrals A, et al. Cross-National Analysis of Associations among Mental Disorders and Suicidal Behavior: Findings from the WHO World Mental Health Surveys. Plos Medicine 2009; 6(8):1-17.

36. Caprara GV, Paciello M, Gerbino M, Cugini C. Individual differences conducive to aggression and violence: Trajectories and correlates of irritability and hostile rumination through adolescence. Aggressive behavior. Italy; 2007; 33: 359-74

37. Friedman RC, Kornfeld DS, Bigger TJ. Psychological problems associated with sleep deprivation in interns. Journal of Medical Education. United States of America; 1973; 48(5): 436-41.

38. Franzen PI, Buysse DJ, Rabinovitz, M, Pollock BG, Lotrich FE. Poor sleep quality predicts onset of either major depression or subsyndromal depression with irritability during interferon-alpha treatment. Psychiatry Research. 2009; 177: 240-245.

39. Salirrosas-Alegría C, Saavedra-Castillo J. Percepción de algunos estilos de crianza y el episodio depresivo en el adulto. Perú: Revista de Neuropsiquiatría. 2014; 77(3):160-66.

40. Anda RF, Felitti VJ, Bremner JD, Walker JD, Whitfield $\mathrm{Ch}$, Perry BD, et al. The enduring effects of abuse and related adverse experiences in childhood: A convergence of evidence from neurobiology and epidemiology. Eur Arch Psychiatry Clin Neurosci. 2006; 256:174-186.

Recibido: 28/03/2019

Aceptado: 31/03/2019 